\title{
Comentarios sobre 597 casos de Presentación de Pelvis
}

\author{
Por el Dr. Roberto A. Páez Vargas \\ Instituto Materno Infantil de Bogotá - 1961 \\ Trabajo presentado para el ascenso a R3
}

\section{INTRODUCCION}

Durante el tiempo de permanencia en el Instituto Materno Infantil, fué de mi atenta observación la angustia que a todos y cada uno de los que hemos pertenecido a la Institución, nos produce el tener obligación de verificar el diagnóstico de una gestación cuyo polo que se presenta es una Pelvis, cualquiera que sea la modalidad.

Hallo la oportunidad de presentar un trabajo, cuya finalidad es primordialmente actualizar en la forma más concisa que me ha sido posible, la incidencia de tal presentación durante los años de 1958 y 59 de acuerdo con la codificación estadística del Instituto, a más de las conductas en los casos complicados o no, para de este modo lograr extraer enseñanzas prácticas para la oportuna atención de un parto en Pelvis, dadas las peculiaridades del mismo, ya que como siempre se ha dicho, es el único en que se verifica realmente el parto de los tres polos en todos y cada uno de sus tiempos.

Para la mejor organización del tema, desarrollaré los siguientes capítulos:

1 - MATERIAL Y METODOS. El material que me ha servido, está constituído por las Historias Clínicas consignadas en el Departamento de Estadística, cuyo contenido siempre lo creemos fehaciente y es de notar, como en otro capítulo se comenta, la debilidad personal de las gestantes en su asistencia oportuna a los servicios prenatales.

2 - En un segundo capítulo me permito analizar y comentar en forma suscinta pero documentado con los conceptos de $\mathrm{Au}$ - 
tores y Profesores tanto antiguos como modernos y ayudado de la experiencia que el continuo trabajo y el deseo de aprender cada día "una enseñanza más", le proporciona al Especialista la Disciplina Hospitalaria.

En estos comentarios, analizo las conductas en forma global tanto de expectativa como intervencionistas; la manera adecuada o no de conducción de los trabajos; la precipitación o demasiada espera en las maniobras de ayuda; en fin, todo aquello que, como decía en principio, se logra extractar de una somera pero cuidadosa revisión de un sinnúmero de Historias-Casos que por cualquier causa etiológica, dan el resultado de una presentación de Pelvis.

3 - En un pequeño párrafo presentaré un RESUMEN.

4 - En un cuarto capítulo presento unas pocas conclusiones de las cuales, son mi deseo, pudieran servir para la unificación de la conducta o conductas probablemente más adecuadas para mejorar el pronóstico materno fetal en tales presentaciones y dejar un concepto muy claro en lo referente a conducción de los trabajos.

Claro está, que no es mi pretención de fijar en el presente bosquejo, normas taxativas, pero sí sería ya bastante satisfactorio, contribuír en medida de mis capacidades a disminuír en lo posible los índices de morbi y mortalidad perinatal fetal.

Sea la oportunidad de agradecer a todos y cada uno de los miembros del personal del Departamento de Estadística, cuya colaboración fué óptima para la realización del presente trabajo.

PRIMER CAPITULO

\section{MATERIAL Y METODOS}

\section{1 - ANALISIS ESTADISTICO}

El material fué tomado de las Historias clínicas correspondientes a las pacientes que ingresaron al Instituto Materno Infantil en el período correspondiente entre Marzo del año de 1958 y Marzo del año de 1960. Se registró durante ese tiempo un total de ingresos de 31.602 pacientes, de las cuales tuvieron parto ya sea a término o prematuro 21.166 pacientes. Las demás fueron hospitalizadas por otras complicaciones. 
En lo que a partos en presentación de Pelvis se refiere tenemos que hubo un total de 597 partos, los cuales ya mejor clasificados los podemos dividir así: Partos en presentación de Pelvis Completa 386 ; partos en presentación de Pelvis Incompleta 187; y partos en presentación de Pelvis no Especificadas 24.

Analizando estas cifras, podremos llegar a la conclusión de que la incidencia o frecuencia total del parto en presentación de Pelvis, en el Instituto fué de $2.83 \%$ del cual corresponden: el $1.828 \%$ a los partos en Pelvis completas y el $0.883 \%$ a los partos en Pelvis incompletas, el excedente o sea el $0.109 \%$ correspondió a los partos en presentación de Pelvis no especificadas.

\section{2 - FRECUENCIA SEGUN LA PARIDAD}

\section{Paridad}

Primíparas

Secundíparas

Multíparas



$\begin{gathered}\text { Pelvis no } \\ \text { Especificada }\end{gathered}$
7
5
12

Totales

Como puede observarse la mayor incidencia del parto en Pelvis se encuentra en las Multiparas dando un porcentaje del $52,79 \%$, sigue en su orden las primiparas con $27,80 \%$ y por último las secundíparas con un $19,41 \%$.

3 - FRECUENCIA DE ACUERDO

CON LA EDAD DE L AGESTACION

Meses
Nueve meses
Ocho meses $1 / 2$
Siete meses $1 / 2$
Siete meses
Seis meses $1 / 2$
Seis meses

Hago notar que aunque en la actualidad esta edad, se manifiesta en semanas, tal dato no figura consignado en el material disponible. El mayor porcentaje se presentó entre las pacientes a término con un $63,82 \%$ de incidencia; en las pacientes con seis a ocho y medio meses de embarazo la incidencia fué menor.

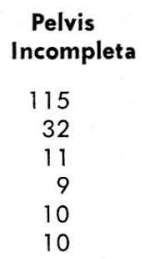



Totales
381
96
40
35
19
26

a ocho y medio meses de embarazo la incidencia fué menor. 


\section{4 - FRECUENCIA SEGUN POSICION Y VARIEDAD DE PRESENTACIONES}

Variedad

SIIA

SIDA

SIIT

SIDT

SIDP

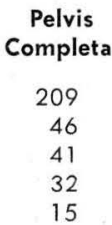

$\begin{array}{cc}\begin{array}{c}\text { Pelvis } \\ \text { Incompleta }\end{array} & \begin{array}{c}\text { Pelvis no } \\ \text { Especificada }\end{array} \\ 93 & 12 \\ 46 & 8 \\ 8 & 3 \\ 13 & 1 \\ 14 & -\end{array}$

Totales

314

100

52

46

29

Quedan 56 casos que no se pudieron incluír en el cuadro anterior, por figurar en la historia solamente la posición, pero no la variedad.

\section{5 - PROMEDIOS DE DURACION DE LOS TRABAJOS DEL PARTO EN RELACION CON LA PARIDAD DE LAS PACIENTES Y EL PESO DEL PRODUCTO DE LA CONCEPCION}

a) PELVIS COMPLETA

Primíparas

Fetos de 1.000 a 2.000 grs.
Fetos de 2.000 a 3.000 grs.
Fetos de 3.000 y más grs.

Multíparas

Fetos de 1.000 a 2.000 grs.

Fetos de 2.000 a 3.000 grs.

Fetos a más de 3.000 grs.

Promedio total de Peso

Fetos de 2.820 gramos

b) PELVIS INCOMPLETA

Primiparas

Fetos de 1.000 a 2.000 grs.

Fetos de 2.000 a 3.000 grs.

Fetos de más de 3.000 grs.

Multíparas

Fetos le 700 a 2.000 grs.

Fetos de 2.000 a 3.000 grs

Metos de más de 3.000 grs.

Promedio Total de Peso

Fetos de 2.517 grs.

No hay marcada diferencia entre primiparas y multiparas
Duración del Trabajo

$10 \mathrm{Hs} .45 \mathrm{mts} .27 \mathrm{sgs}$

$11 \mathrm{Hs} .52 \mathrm{mts}$. 56 sgs.

$10 \mathrm{Hs} .25 \mathrm{mts} .25 \mathrm{sgs}$.

Duración del Trabajo

$9 \mathrm{Hs} .11 \mathrm{mts} .52 \mathrm{sgs}$

$6 \mathrm{Hs} .21 \mathrm{mts} .41 \mathrm{sgs}$

$9 \mathrm{Hs} .22 \mathrm{mts} .48 \mathrm{sgs}$

Promedio total de

Duración del Trabajo

$9 \mathrm{Hs} .40 \mathrm{mts} .01 \mathrm{sgs}$.

Duración del Trabajo

$10 \mathrm{Hs} .5 \mathrm{mts} .0 \mathrm{sgs}$.

$12 \mathrm{Hs} .18 \mathrm{mts} .36$ sgs.

$13 \mathrm{Hs} .23 \mathrm{mts}$. 0 sgs.

Duración del Trabajo

$7 \mathrm{Hs} .14 \mathrm{mts} .17$ sgs.

$11 \mathrm{Hs} .19 \mathrm{mts} .0 \mathrm{sgs}$

$11 \mathrm{Hs} .36 \mathrm{mts}$. 55 sgs.

Promedio Total de Duración del Trabajo

$10 \mathrm{Hs} .59 \mathrm{mts} .28$ sgs. 
tanto en presentación de Pelvis Completa como Incompleta en cuanto a duración del trabajo se refiere, ni tampoco existe una variación muy marcada entre los promedios de duración del parto de las Pelvis Completas y las Incompletas notándose apenas una diferencia de 1 hora 19 minutos 27 segundos de mayor duración en las Pelvis Incompletas.

\section{6 - FRECUENCIAS Y TIPOS DE PARTO SEGUN SU CLASIFICACION}

a) PELVIS COMPLETA

\section{Tipo de Parto}

Espontáneos

Auxiliados y corregidos

OPERATORIOS :

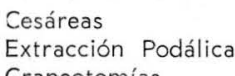

Craneotomías

Tipo de Parto

Espontáneos

Auxiliados y corregidos

OPERATORIOS :

Cesáreas

Fórceps

Tipo de Parto

Auxiliados y corregidos

OPERATORIOS :

Cesáreas
Extracción Podálica
Extracción Podálica

Espontáneos

\section{Primíparas}

46

36

13

1

b) PELVIS INCOMPLETA

Primíparas

\section{Multíparas}

Total

21

29

80

41

101

70



c) PELVIS NO ESPECIFICADAS

Primíparas
3
2

\section{Multíparas}

Total

He hecho la clasificación de los tipos de partos como aparece en los cuadros anteriores en:

ESPONTANEOS: O sean aquellos en donde el Obstetra no tiene nada que hacer solo el papel pasivo de recibirlo, como en cualquier parto, en presentación de vértice y pequeñas maniobras tales como episiotomías, elevación del tronco o sostenimiento del mismo con una compresa que por la rutina de las mismas no se consideran como intervención. 
AUXILIADOS Y CORREGIDOS: O sean aquellos en que el Obstetra ayuda a la conducción del parto, mediante maniobras, ya sea en ayuda a un parto normal con el fin de acelerar su expulsión, o para corregirlo por alguna complicación que surja en el transcurso de éste. En este grupo están comprendidas las maniobras de MAURICEAU, CHAMPETIER DE RIBES, ROJAS y otras maniobras correctoras de la deflexión de la cabeza última.

PARTOS OPERATORIOS: O sean aquellos en que haya necesidad de intervenir, ya sea quirúrgicamente mediante CESAREA o por extracción podálica; con operaciones mutilantes para el feto tales como craneocentésis; o en último caso mediante aplicación de fórceps para cabeza última. Debe anotarse que la mayoría de los partos fueron espontáneos llegando al $57,17 \%$ en Pelvis Completa y al $54,01 \%$ en Pelvis Incompleta.

El porcentaje de partos auxiliados y corregidos fué del $30,31 \%$, para Pelvis Completas y del $37,43 \%$ para Pelvis Incompletas.

En cuanto a Cesárea se refiere hubo un $7,03 \%$ para el parto en Pelvis en general. Para el parto de Pelvis Completa el porcentaje fué de $7,51 \%$; y para la Pelvis Incompleta el porcentaje de cesáreas fue de $4,81 \%$.

Al relacionar con el número total de cesáreas efectuadas en el Instituto durante el mismo tiempo de este trabajo por diversas indicaciones cuyo total fué de 991 cesáreas, el porcentaje para Pelvis en general corresponde al 4,24\%.

\section{7 - MORTALIDAD PERINATAL $Y$ SUS DIFERENTES CAUSAS}

a) PELVIS INCOMPLETA

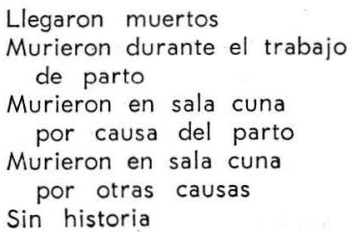

Primíparas
3
6
3
2
3

\begin{tabular}{cc} 
Multíparas & Total \\
12 & 15 \\
6 & 12 \\
6 & 9 \\
9 & 11 \\
- & 3 \\
\hline
\end{tabular}




\section{CAUSAS DE LA MUERTE FETAL}

MURIERON DURANTE EL TRABAJO DE PARTO

MURIERON EN SALA CUNA POR CAUSA DE PARTO

MURIERON EN SALA CUNA POR OTRAS CAUSAS

\section{Primíparas}

\section{Multíparas}

5 Retención de cabeza

1 Placenta Previa

3 Retención de cabeza

3 Placenta previa

1 Anoxia por retención de cabeza

1 Anoxia por extracción podálica

Neumonitis
1 Prematurez y placenta previa

1 Anemia aguda por ruptura del piso de la boca

1 Extracción podálica por procidencia del cordón

1 Inmadurez

1 Gemelar Hist. N6 69753

1 Trauma hepático y onfanocele

1 Malformaciones congénitas. 1 Sepsis Cardiopatía

1 Prematurez y sepsis 1 Prematurez y bronconeumonía hemorrágica 1 Anomalías congénitas

1 Gemelar prematuro y sepsis

1 Enfermedad hemorrágica y prematurez Prematurez

Sepsis

1 Prematuro y anemia por placenta previa

Total de fetos muertos de los partos en PELVIS INCOMPLETA

b) PELVIS COMPLETA

$\begin{array}{lccc} & \text { Primíparas } & \text { Multíparas } & \text { Total } \\ \text { Llegaron muertos } & 7 & 26 & 33 \\ \begin{array}{l}\text { Muertos durante el trabajo de parto } \\ \text { Muertos en sala cuna }\end{array} & 4 & 13 & 17 \\ \quad \text { por causa del parto } & 5 & 12 & 17 \\ \begin{array}{l}\text { Muertos en sala cuna } \\ \text { por otras causas }\end{array} & 5 & 23 & 28 \\ \text { Sin historia } & - & 1 & 1\end{array}$

\section{CAUSAS DE LA MUERTE FETAL}

MUERTOS DURANTE

$$
\text { Primíparas }
$$

EL TRABAJO DE PARTO

\section{Multíparas}

2 Retención de cabeza
1 Procidencia del cordón
1 Eclampsia

7 Retención de cabeza

2 Placenta previa

1 Hipertonía uterina y procidencia del cordón

1 Inmadurez

1 Prematurez y lúes

1 Lúes Hepatomegalia 
MUERTOS EN SALACUNA POR CAUSA DEL PARTO

MUERTOS EN SALA CUNA POR OTRAS CAUSAS

\section{Hemorragia intracraneal \\ 1 Neumonía aspirativa, y labio leporino \\ 1 Anoxia por trauma obstétrico \\ 1 Sufrimiento encefálico}

3 Prematuros con mala ventilación pulmonar

1 Esclerema y anoxia
3 Hemorragia intracraneal

2 Trauma Obstétrico y ventilación pulmonar anormal

1 Anoxia por ventilación pulmonar anormal

1 Anoxia por placenta previa

1 Retención de cabeza $p$ bronconeumonía

1 Colapso cardiovascular

1 Neumonía aspirativa y prematurez

1 Sufrimiento encefálico por parto rápido

6 Prematuros con mala ventilación pulmonar

3 Malformaciones congénitas

4 Sepsis

3 Neumonías hemorrágicas

2 Eritroblastosis

1. Prematuro hemopatía e insuficiencia suprarrenal

1 Neumonía y sepsis

1 Colapso pulmonar

1 Membrana hialina

1 Indeterminado

Total de fetos muertos en partos de PELVIS COMPLETAS

Es de anotar que fuera de estos casos hay 44 más que corresponden: 11 a PELVIS INCOMPLETA y 33 a PELVIS COM$P L E T A$, de los cuales no se sabe si el feto salió o no vivo del Instituto, ya que faltan las historias pediátricas.

\section{c) PELVIS NO ESPECIFICADAS}

\begin{tabular}{|c|c|c|c|}
\hline & Primíparas & Multíparas & Total \\
\hline Llegaron muertos & 2 & 5 & 7 \\
\hline $\begin{array}{l}\text { Murieron durante el trabajo de parto } \\
\text { Murieron en sala cuna } \\
\text { Murieron en sala cuna }\end{array}$ & 一 & - & - \\
\hline por causa del parto & 3 & - & 3 \\
\hline por otras causas & - & 2 & 2 \\
\hline
\end{tabular}

CAUSAS DE LA MUERTE FETAL

Primíparas

MURIERON EN SALA CUNA POR CAUSA DEL PARTO
Multíparas

1 Hemorragia endocraneana 
MURIERON EN SALA CUNA

POR CAUSA DEL PARTO

MURIERON EN SALA CUNA POR OTRAS CAUSAS
1 Hemorragia y menín-

gea y prematurez

Prematuro y sufrimiento

encefálico
1 Sepsis, bronconeumonía y cardiopatía

1 Prematurez $y$ atelectasia pulmonar

Total de fetos muertos correspondientes a los partos en PELVIS NO ESPECIFICADAS 12

Del estudio de los cuadros anteriores, podemos deducir: que la mortalidad global fetal por partos en presentación de $P E L$ VIS EN GENERAL, es del $26,46 \%$.

La mortalidad global fetal por partos en presentación de PELVIS INCOMPLETA, es del 26,74\%.

La mortalidad global fetal por partos en presentación de PELVIS COMPLETA, es del 24,87\%.

Depurando la mortalidad fetal, hasta tanto no tener en cuenta para el porcentaje, sino solo aquellos casos de mortalidad por causas obstétricas; encontramos que nos dá un porcentaje para la Pelvis Incompleta del 9,09\%; y para la Pelvis Completa del $6,73 \%$.

\section{8 - CESAREAS EFECTUADAS EN EL INSTITUTO DURANTE ESTE MISMO TIEMPO, EN LOS CASOS DE PARTO EN PRESENTACION DE PELVIS $Y S U I N D I C A C I O N$}

Indicaciones
Primigestante añosa
Placenta previa
Procúbito del cordón
Cesáreas iterativas
Inercia uterina secundaria
Distocia de cuello
Distocia de partes blandas
Infección amniótica
TOTAL DE CESAREAS ..........

Casos
3
7
2
6
1
2
1
2

\section{Indicaciones}

Procidencia del cordón

Sufrimiento fetal agudo

Estrechez pélvica

Por feto grande

Muerte habitual del feto

Desproporción cefalopélvica

Parto mal conducido

MUERTES FETALES . . . . . . . . . 6

La mortalidad fetal en los partos por cesáreas fué de 6 casos, los cuales corresponden así: 2 que llegaron muertos por placenta previa central total, 2 que murieron por malformaciones 
congénitas; y 2 que murieron durante el trabajo de parto: 1 por placenta previa central total (anoxia) y otro por taquisistolia uterina y procidencia del cordón.

\section{9 - EXTRACCIONES PODALICAS EFECTUADAS}

Se efectuaron durante ese mismo tiempo 25 extracciones podálicas, de las cuales 9 fueron con resultados satisfactorios; 9 con fetos muertos así: 3 que murieron en sala cuna por causa de la intervención (anoxia, prematurez y ventilación pulmonar anormal) 3 que liegaron al Instituto con feto muerto y macerado; 2 que murieron en sala de partos, por retención de cabeza última por falta de dilatación completa del cuello; y uno que murió por anoxia durante el trabajo de parto, por haber hecho la paciente un ataque de eclampsia.

De los 7 casos restantes se desconoce la suerte final de los fetos, por no aparecer las historias pediátricas correspondientes.

El porcentaje de extracciones podálicas hechas en el Instituto fué del $41,19 \%$ para presentación de pelvis en general. La mortalidad depurada, por esta intervención fue del $20 \%$.

\section{0 - MORTALIDAD PERINATAL COMPARATIVA SEGUN EL TIPO DE PARTO}

Tipo le parto

Espontáneos

Auxiliados y Corregidos

OPERATORIOS :

Cesáreas

Extracción Podálica

Sin historia 4

$\begin{array}{rrrr}\begin{array}{c}\text { Fetos que } \\ \text { Ilegaron } \\ \text { tos }\end{array} & \begin{array}{c}\text { Murieron du- } \\ \text { rante el } \\ \text { trabajo de parto }\end{array} & \begin{array}{c}\text { Muertos en } \\ \text { sala cuna por } \\ \text { causa del parto }\end{array} & \begin{array}{c}\text { Muertos en } \\ \text { sala cuna por } \\ \text { otras causas }\end{array} \\ 40 & 3 & 19 & 28 \\ 11 & 20 & 7 & 11 \\ 2 & 2 & - & 2 \\ 2 & 4 & 3 & -\end{array}$

De este cuadro se deduce que la mortalidad global fetal en partos espontáneos (incluyendo los fetos que llegaron muertos) fué del $26,78 \%$; de los cuales solo el $6,55 \%$ corresponden a muertos por causas obstétricas puras.

En los partos Auxiliados y Corregidos, la mortalidad global fetal fué del 25,52\% (incluyendo los fetos que llegaron muertos); de los cuales el $14,06 \%$ corresponde a muertos por causas obstétricas puras. Debe anotarse que en este tipo de partos, la mortalidad asciende considerablemente, por causas obstétricas puras, 
de donde puede deducirse que las complicaciones y maniobras prematuras en la expulsión de los hombros y la cabeza, elevan notoriamente la mortalidad, desafortunadamente en las historias, no está claramente especificada, si las maniobras fueron hechas para auxiliar un parto complicado, o si las maniobras se hicieron prematuramente complicando de esta manera el parto.

En cuanto a Cesáreas la mortalidad disminuye por causa obstétrica prácticamente al $0 \%$, aún considerando que las indicaciones fueron en la mayoría de los casos de otra índole y no se encuentra ninguna indicación de cesárea por el solo hecho de tratarse de un parto de pelvis, a excepción quizás de 3 casos de primigestante añosa.

En cuanto a Extracción Podálica se refiere, el comentario ya fue hecho en el cuadro número 9 .

\section{CAPITULO SEGUNDO}

\section{COMENTARIOS SOBRE CONDUCTA Y PRONOSTICO DEL PARTO EN PRESENTACION DE PELVIS}

En este Capítulo presento en primer término: cuál es el concepto sobre la conducta y pronóstico en el parto en presentación de Pelvis, dada por los principales Autores de Obstetricia, los Profesores de Obstetricia de la Universidad Nacional y algunos Instructores del Departamento Obstétrico del Instituto Materno Infantil.

A continuación presentaré unos comentarios personales, basados en la experiencia y enseñanza que he adquirido durante mi permanencia en el Instituto Materno Infantil y lo que he logrado extractar después de una somera pero cuidadosa revisión de 597 historias-casos de partos en presentación de pelvis.

1 - JUAN LEON : En su tratado de Obstetricia dice:

\section{A) PRONOSTICO DEL PARTO PELVIANO}

En términos generales el pronóstico del parto pelviano, tanto el materno como el fetal, es menos favorable que el del parto en presentación de vértice.

1 - Como bien dice V. DONATO: El pronóstico materno es grave solo cuando se practican intervenciones intempestivas, en 
particular la gran extracción con dilatación incompleta, con los riesgos de desgarros del cuello y del segmento inferior.

2 - Pronóstico para el feto: Se considera en general que el pronóstico fetal es desfavorable, por cuanto el parto de los hombros (con la posibilidad del levantamiento de los brazos) y el de la cabeza, constituyen siempre una amenaza.

La mortalidad fetal global (no depurada) es alta en algunas estadísticas $(20 \%)$ o más; mediana en otras $(10 \%$ a $20 \%)$ y aun baja (menos de 10\%).

La mortalidad fetal depurada solo considera la muerte atribuíble a la clase de presentación, pura y exclusivamente al parto pelviano a las maniobras utilizadas en la atención del mismo y a sus consecuencias ulteriores. La mortalidad fetal corregida es alta, por pasar de $10 \%$. Algunos Autores han registrado una mortalidad fetal corregida que oscila entre 2 y $10 \%$. Por fin, en algunos ambientes la mortalidad fetal corregida es halagüeña, pues, está por debajo del $2 \%$.

La morbilidad fetal asociada con la presentación pelviana es de trascendencia, ya que muchas lesiones son serias y permanentes. Dichas lesiones son acaso determinadas por las maniobras extractoras. En cambio en los partos pelvianos espontáneos, la morbilidad fetal es casi nula.

\section{B) CONDUCTA ANTE UNA PRESENTACION PELVIANA}

1 - Conducta en el embarazo: Durante el embarazo, es preciso efectuar un examen prolijo de la paciente.

Establecer el diagnóstico de la presentación pelviana, de su variedad y modalidad y cerciorarse mediante la auscultación de que el feto está vivo.

Realizar un esmerado examen clínico de la pelvis de toda mujer cuyo feto se halla en presentación podálica, que se complementará con un examen radiológico cuando sea necesario, sobre todo en las primíparas y con más razón en las primíparas añosas y en las multíparas con antecedentes de partos dificultosos.

Apreciar lo más exactamente posible el tamaño del feto.

En vista de que el parto pelviano tiene, en términos generales, un pronóstico más desfavorable que el parto en presenta- 
ción de vértice, es lógico tentar la transformación de la presentación pelviana en una cefálica en las últimas semanas de la gestación.

Otra manera de efectuar la profilaxis del parto pelviano consiste en practicar la cesárea abdominal antes de la iniciación del parto (Cesárea de elección).

En la presentación pelviana, se realiza con una frecuencia variable la cesárea abdominal de elección (0,5 a más de $25 \%)$. En nuestro servicio del Hospital Argerich, la incidencia de cesáreas en la presentación pelviana fué de 2,6\% (J. León y Azulay).

A nuestro juicio, la presentación pelviana en sí no puede ser considerada como una indicación para la intervención; ésta se plantea únicamente cuando, después de un examen detenido, se descubren una o varias circunstancias que hacen sospechar dificultades en el parto por las vías naturales para la madre o el feto, o para ambos.

2 - Conducta en el parto: Si durante el embarazo no se ha logrado transformar la presentación pelviana en una cefálica y si no se ha recurrido a una cesárea abdominal "electiva", quedan, una vez ya en marcha el parto pelviano, tres eventualidades:

a) Ejecutar la versión externa.

b) Efectuar la cesárea abdominal.

c) Dejar que el parto evolucione por las vías naturales.

La versión externa, es poco prudente realizarla, por el riesgo de que se transforme la presentación pelviana en otra más distócica. Con las membranas rotas, está contraindicada, ya que surge el peligro de la procidencia del cordón.

La cesárea abdominal, en un parto pelviano ya iniciado, tiene indicaciones absolutas:

a) Cuando se comprueban signos clínicos o radiológicos de una marcada desproporción feto-materna, de un tumor previo, de una inserción baja de la placenta, etc.

b) En presencia de ciertos factores intercurrentes (distocia uterina, dilatación estacionaria del cuello, procidencia del cordón con escasa dilatación, sufrimiento fetal también con dilatación incompleta, etc.). 
Las indicaciones relativas de la cesárea abdominal las constituyen la peivis "límite", la primiparidad añosa, resultando difícil en tales casos tomar una decisión.

El parto por las vías naturales del feto en presentación pelviana puede ser, de acuerdo con la conducta que se sigue en su atención: a) parto pelviano espontáneo. b) parto pelviano con ayuda manual. c) Parto pelviano operatorio (se emplea este término cuando se extrae el cuerpo entero del feto, inclusive el polo pelviano.

En la mayoría de los casos, cuanto menos intervenga el Médico y cuanto más tardíamente lo haga -sobre todo cuando se trata de un Médico con limitada experiencia- tanto mejor para la seguridad de la madre o del niño.

Mueren más fetos por lesiones encefálicas provocadas por ese apresuramiento, que por anoxia debida al paso lento a través del canal de parto.

2 - EASTMAN : dice en su tratado OBSTETRICIA DE WILLIAMS:

"Pronóstico: Con respecto a la parturienta hay muy poca diferencia entre el pronóstico de las presentaciones de nalgas y el de las de vértice. En cambio, con respecto al feto el pronóstico es mucho más desfavorable que en las presentaciones de vértice, sobre todo cuando el feto es grande y la mujer es primigrávida.

La letalidad fetal total, incluyendo los partos prematuros, los de término y los fallecimientos en el período neonatal, es aproximadamente del $20 \%$.

TRATAMIENTO. En vista del grave pronóstico con respecto al feto en las presentaciones de nalgas debe siempre el partero tratar de evitarlas en cuanto sea posible y siempre que las reconozca en las últimas semanas de la gestación procurará convertirlas en presentaciones de vértice por medio de la versión externa, procedimiento que si se ejecuta debidamente y con delicadeza acarrea poco peligro, aunque con ella no se logra todo lo que sería de esperar. Se puede también ensayar esta en el primer período del parto, siempre y cuando no hayan descendido profundamente las nalgas en la pelvis, pues una vez que se fijan es inútil todo esfuerzo. 
En la mayoría de las presentaciones de nalgas se efectúa la expulsión espontánea hasta el ombligo, y de ahí en adelante debe el tocólogo concretarse a observar el parto y esperar, pero estará siempre listo para intervenir en cualquier momento. Por esta razón, tan pronto como las nalgas aparezcan en la vulva se prepara a la paciente para la expulsión a efecto de que no se pierda ni un segundo en la extracción, si ésta fuere necesaria. Se dejará que las nalgas avancen espontáneamente hasta que salga el ombligo. Es de suma importancia recordar que se facilita mucho la expulsión cuando los brazos conservan su posición normal cruzada sobre el tórax y cuando la cabeza está en flexión extrema. Para lograr esto se evitará cuanto sea posible la tracción, así como la presión sobre el fondo que, según dijo Potter, pudiera empujar la cabeza entre los brazos.

En vista de la crecida letalidad fetal, Piper sostiene que muchas criaturas mueren como consecuencia de la extracción en cuyo lugar recomienda la aplicación de fórceps a la cabeza última".

3 - HENRI VERMELIN y MARCEL RIBON dicen: En la Enciclopedia Médico Quirúrgica 1er. Tomo Obstétrico Pág. 5026 A20 año 1952.

“Elementos de pronóstico e indicaciones generaless

La opinión clásica dá un pronóstico más reservado para la pelvis incompleta, porque produce más fácilmente ascenso de los brazos y dificultades en el encajamiento.

Conducta. Es necesario evitar intervención en el período de expulsión y solo hacerlo en los casos precisos.

Aconseja durante el periodo de la gestación la versión a cefálica en el séptimo mes en primíparas y en el octavo mes en multíparas.

Durante el periodo de dilatación. La distocia dinámica adquiere en las presentaciones pélvicas una importancia primordial.

Conducta en el periodo de expulsión. Cuando no existe ninguna urgencia de extracción del feto el parto debe ser espontáneo, pero si existe alguna urgencia materna o fetal para la terminación del parto, debe hacerse la extracción podálica".

4 - NUBEOLA-ZARATE (Tratado de Obstetricia Tomo I)

"En principio: 
La presentación de nalgas incompleta en primípara es sinónimo de indicación de extracción manual. Es ésta la indicación específica de extracción manual, ejecutándose a su debido tiempo, si no existen circunstancias que la anulen, como, por ejemplo: Pelvis impracticable".

5 - CONCEPTO PROFESOR RAFAEL RAMIREZ MERCHAN : (Comunicación personal).

"Presentación de Pelvis - Conducta - Pronóstico:

Auncuando se acostumbra hacer diferencia entre la pelvis completa y la incompleta, para efecto de la conducta obstétrica, en términos generales, la conducta obedece a las mismas normas; solamente es de advertir que puede sobrellevar mayores complicaciones la pelvis incompleta que la completa.

También debe tenerse muy en cuenta en las consideraciones que determinan una u otra conducta la primi o multiparidad de la paciente.

Un estudio muy cuidadoso de las condiciones anatómicas y funcionales de la madre así como también sus condiciones obstétricas en relación con capacidad pelviana y ausencia o presencia de complicaciones obstétricas como placenta previa $\mathrm{u}$ otras $\mathrm{y}$ aspectos relativamente nuevos como Factor $R h$ debe proceder a la decisión de una conducta.

Un conocimiento a detalle del mecanismo del parto en dicha presentación es indispensable cuando se asiste el parto espontáneo o forzosamente conducido en algunos de sus períodos.

De todas maneras, el parto por las vías naturales tiene un índice de mortalidad infantil que oscila entre el 6 y $9 \%$. Pero la incidencia general, en casos no complicados, "es inversamente proporcional a la habilidad del partero". En forma tal que los índices pueden bajar a un 2,7\% según autoridades en la materia.

Una vez estudiada debidamente la paciente, la conducta es:

1 - Parto espontáneo.

2 - Parto intervenido con maniobras manuales; o fórceps en cabeza última.

3 - Cesárea. 
1 - La Cesárea es de indicación imprescindible en los casos:

a) De pelvis estrecha o límite.

b) En primigestante vieja.

c) En primigestante $\mathrm{Rh}(-)$ negativa.

d) En feto grande, en que se juzgue que el tamaño mismo ha decidido la presentación de pelvis.

e) Desde luego, cuando ocurre el sufrimiento fetal y no está cercana la expulsión ni indicada la extracción de pelvis.

f) Cuando se prolonga el trabajo del parto por distocia dinámica con cuello de dilatación estacionaria.

g) Finalmente cuando hay razón para practicar una cesárea o por causa distinta a la presentación.

La incidencia de la cesárea en esta presentación es del 13 al $45 \%$.

PARTO POR LAS VIAS NATURALES:

1 - Parto espontáneo: Es excepcionalmente raro que ocurra sin intervención alguna pero éste puede hacerse en casos especiales que sorprenden a quien tiene la vigilancia del caso.

2 - Parto intervenido: Con maniobras manuales o fórceps en cabeza última: Cuando se descarta la cesárea y se decide el parto por las vías naturales deben extremarse los cuidados con la paciente para impedir las complicaciones así:

a) Reposo y sedación.

b) Mantenerla acostada para propiciar la integridad de la bolsa.

c) Evacuación de recto y vejiga.

d) Hidratación por vía parenteral.

e) Evitar exámenes vaginales innecesarios con el fin de no edematizar el cuello y mantener integridad de las membranas.

f) Esperar a la dilatación completa del cuello y a que desaparezca de vagina antes de aconsejar la ayuda materna con los esfuerzos expulsivos.

g) Un vez iniciada la expulsión prescindir de toda tracción, dejando la expulsión a la "vis a tergo". 
h) Si se considera que se necesita ayuda hacerlo con presión sobre el fondo uterino.

i) Iniciada la expulsión, conducirla de acuerdo con el mecanismo normal del parto. Insistir mucho en ayudar el dorso a que se haga anterior y no traccionar.

j) Una vez expulsado el polo pélvico y aparecido el cordón hacer una asa para evitar que se presione.

k) Aumentar en este momento la presión del ayudante sobre el fondo uterino para mantener la cabeza en flexión y propiciar su encajamiento.

1) Proceder a la extracción de los brazos. Si no se ha hecho uso de la "Vis a fronte" de seguro que no se han ascendido y es fácil su extracción.

m) Proceder luego a ayudar a la extracción de la cabeza con la maniobra de Mariceau. Siempre debe utilizarse la presión del ayudante.

n) La maniobra tiene cinco tiempos:

1 - Búsqueda de la boca y colocación de los dedos međio e índice dentro de la misma; la mano libre sobre los hombros y la nuca.

2 - Flexionar la cabeza lógicamente, con suaves tracciones sobre la boca y empujando el occipital con la mano que se colocó allí.

3 - Completar la rotación a OP.

4 - Descender.

5 - Proceder a la extracción.

Terminación con Fórceps:

Algunos prefieren usar sistemáticamente el Fórceps y prescindir de las maniobras manuales; en tal caso el Fórceps más apropiado es el F. de Piper.

Personalmente prefiero las maniobras manuales porque considero que practicadas correctamente son más oportunas y menos traumatizantes que el fórceps. El ejercicio particular de la especialidad me ha llevado a este convencimiento. 
Pero cuando el caso no ha sido bien conducido o cuando a pesar de una correcta "no intervención extemporánea" la cabeza se defleja o no rota a OP o ha rotado a OS entonces es de indicación forzosa el citado instrumento. En tales casos ya hay una amenaza cierta para la vitalidad fetal.

Son esos los casos que unidos a los de procidencia del cordón ascenso de los brazos dan los índices de mortalidad ya citados.

Extracción Podálica: Cuando lograda la dilatación completa la presentación en cuestión queda estacionaria o no se encaja u ocurre sufrimiento fetal está indicada la extracción de pelvis; empero, esta intervención contraría la norma fundamental del éxito que es la no intervención extemporánea en relación con los distintos tiempos del parto. Por eso es preferible optar por la cesárea en tales casos; todo en beneficio de la integridad fetal. Pero si las circunstancias obligan tal intervención entonces debe procederse con la asesoría de un ayudante que no descuidará mantener la presión sobre el fondo uterino, y las tracciones deben practicarse durante la contracción uterina. El ascenso de los brazos, la deflexión de la cabeza, su defecto de rotación o su rotación a sacra son los escollos que no siempre logran evitarse, y una vez producidos, las maniobras ideadas para resolver la situación traumatizan al feto en forma irreparable.

En Resumen: La presentación de pelvis exige riguroso estudio y acertada conducción y aún da un índice alto de intervenciones y un porcentaje no despreciable de mortalidad fetal".

6 - PROFESOR RAFAEL PERALTA CAYON (Comunicación personal).

"Conducta en el parto en Presentación de Pelvis: Hay dos elementos que se deben tener en cuenta y son la dinámica uterina y el cuello. En cuanto a dinámica que sea normal y progresiva; respecto al cuello, que no sea un cuello problema, a fin de que los tiempos fundamentales del parto no tengan contratiempo y entonces haya encajamiento perfecto y en el momento de la expulsión, no haya la menor duda de que la dilatación cervical sea completa y entonces, se evite la retención de cabeza última, o la extracción con Moriceau estando el cuello incompletamente dilatado. En este sentido último, no obstante que algunos la critiquen, la ayuda que ofrezca otro especialista con la maniobra de 
Cristeller bien conducida, es definitiva para la expulsión final de la cabeza y personalmente la prefiero al Fórceps.

7 - PROFESOR SANTIAGO LLERAS CODAZZI. (Comunicación personal).

"Conducta en el parto en presentación de Pelvis: El parto en presentación de pelvis está en el límite de lo normal y lo distócico, hay que tener en cuenta múltiples factores, tales como paridad, edad, antecedentes obstétricos y evaluación de pelvis y cabeza. Cuando se trata de primípara añosa la conducta es cesárea. Pacientes con antecedentes de cesárea, se hace cesárea para evitar rupturas uterinas".

8 - DOCTOR JESUS GOMEZ PALACINO. Asistente de Cátedra. Comunicación personal.

El parto en presentación de pelvis pone a prueba la experiencia del Obstetra porque exige no solamente un juicio clínico previo muy completo sino que demanda una observación permanente de la evolución del parto. Varios elementos vale la pena tener en cuenta: dinámica eficiente que se traduzca en brevedad en el tiempo (menor que en una presentación de vértice), conducción adecuada empleando juiciosamente los ocitócicos, analgesia apropiada en el momento del parto, en mi concepto utilizando el Trilene que sin disminuír a la contractilidad uterina permita la colaboración de la paciente y facilita la intervención manual o instrumental del Obstetra. A pesar de cumplir estrictamente los preceptos enunciados la morbi-mortalidad fetal en el parto en presentación de pelvis es alta, de donde se deduce la posibilidad de aumentar la frecuencia en la intervención cesárea, con miras a mejorar el pronóstico. En el medio hospitalario es recomendable que el manejo de estos casos esté en las manos más experimentadas".

9 - DOCTOR FERNANDO SANCHEZ TORRES. Instructor. Comunicación Personal.

"PRONOSTICO Y CONDUCTA EN EL PARTO EN PELVIS".

"El pronóstico del parto en presentación de pelvis, cualquiera que sea su modalidad, depende exclusivamente de tres factores:

1 - Del correcto juicio con que se valore cada caso en par- 
ticular. Tendremos en cuenta para ello los antecedentes maternos (por ejemplo Priamiañosidad, cesárea previa, etc.) y las características del embarazo actual en el instante de iniciarse el trabajo de parto (Por ejemplo edad gestacional, relación fetopélvica, estado del canal del parto, etc.).

2 - De la adecuada conducción del parto. Se tendrán en cuenta entre otras los siguientes aspectos: sedación de la paciente, dinámica uterina de óptima calidad, delicadas y escasas exploraciones del cuello uterino, integridad de las membranas en el parto prematuro, anestesia que no prive de la conciencia a la parturienta (por ejemplo en silla de montar) ya que en la presión intra-abdominal voluntaria, sumada a la contracciỏn uterina, radica el éxito del período expulsivo.

3 - De no hacer nada distinto a observar, en actitud alerta, el mecanismo natural durante la fase expulsiva.

La CONDUCTA. Depende, pues, fundamentalmente del primer factor. Un recto criterio clínico tendrá que señalarnos oportunamente la vía vaginal o la vía abdominal”.

10 - HERBERT F. SCHMITZ M.D., CHARLES J. SMITH, M.D., EUGENE R. CHUMPNER. Chicago, Ill.

\section{PARTO EN PRESENTACION DE PELVIS.}

La frecuencia del parto en presentación de pelvis es del 3 a $4,8 \%$.

La influencia de la episiotomía sobre la Mortalidad y Morbilidad infantil:

Muchos autores consideran que, debe establecerse el uso de la episiotomía, necesariamente para protección fetal. Su opinión es que el daño cerebral disminuye grandemente con el uso de la episiotomía. Barnel y Bill en un artículo de discusión de Potter, dice que la episiotomía es salvadora para el feto y es obligatoria en todos los partos de pelvis. Comparando estadísticas entre partos atendidos con episiotomía y partos atendidos sin ella, la mortalidad fetal ha llegado a más del doble en los segundos.

Entre sus conclusiones dice: la extracción podálica causa la más alta morbilidad fetal y puerperal y aumenta dos veces más del número de hemorragias postpartum. 
Parto con trabajo prolongado y con extracción podálica, acarrea mortalidad fetal mucho más alta que en los espontáneos.

\section{CONCLUSIONES :}

1 - Evolución espontánea del parto hasta el omoplato y ombligo.

2 - Anestesia profunda.

3 - Observación atenta y tomar las medidas necesarias para mantener la vitalidad materna y fetal en el trabajo prolongado.

4 - Uso de la gran episiotomía en todos los partos de pelvis.

11 - KNIGHT AND O CONNELL del Hospital para mujeres. New York N.Y.

La frecuencia en el parto en presentación de pelvis es del $3,86 \%$.

La versión externa es la conducta en este Hospital y durante este año un total de 368 versiones se efectuaron, cuyos resultados fueron los siguientes: 277 pacientes o sea el 75,3\% terminaron con parto en presentación de vértice.

69 pacientes, o sea el $18,7 \%$ terminaron con parto en pelvis.

22 pacientes, o sea el $6 \%$ con otros tipos de parto, entre ellos partos intervenidos con cesáreas. Entre todos estos casos se presentaron solo 8 de muerte fetal, por diversas causas.

\section{COMENTARIOS}

Al intentar analizar la conducta en la atención de los partos de pelvis de acuerdo a nuestras estadísticas locales, encontramos como primera medida la dificultad del análisis completo de las historias por carencia en la mayoría de ellas de datos explicativos de las maniobras, debido a falla disciplinaria en la elaboración de las mismas. La estadística global que se presenta se halla también notoriamente afectada por descuidos extra hospitalarios, ya que tan solo del $5 \%$ de las pacientes hay constancia de que hubieran asistido a prenatal, la gran mayoría ingresaron en trabajo de parto avanzado, con descuido notorio en las normas higiénicas y profilácticas, de allí la alta incidencia de fetos muertos ingresados al servicio. 
Por otra parte ha existido en el Instituto la enorme falla del reconocimiento de jerarquías dentro del personal asistencial, lo que trae consigo una inadecuada atención del parto, de allí la enorme incidencia de parto auxiliados y corregidos sin discriminación especial del momento y del caso. Se nota que la mortalidad fetal por causa obstétrica se encuentra entre casos atendidos aún por el Jefe de Turno, pero es de anotar que estos muy posiblemente han sido notificados después de varios minutos de complicación y después de que el Interno ha fallido en su intento de extraer el feto. Sinembargo la mortalidad no es susceptible de disculpas de ninguna clase y para disminuírla será necesario hacer efectiva y rígida una disciplina hospitalaria con obligación de dar aviso inmediato al Jefe de Turno, cada vez que llegue una paciente con presentación podálica.

Es fácil deducir de estas estadísticas que el parto en pelvis es altamente peligroso y que la conducción del mismo depende de numerosos factores y de una cuidadoso análisis del estado general de la paciente, apreciación de la pelvis, tamaño del feto y una cuidadosa observación de la dinámica uterina; sin estos requisitos cualquier intento de atención del parto de pelvis es más que lesiva para el futuro del feto.

Haciendo estas salvedades y analizando rudamente las estadísticas encontramos una mortalidad muy alta que corresponde al $6,73 \%$ en pelvis completas y al $9,09 \%$ en incompletas la cual me permito comparar con otras estadísticas:

\section{1 - KNIGHT AND O'CONNELL :}

Mortalidad global fetal en partos de pelvis 20,4\%. Mortalidad fetal corregida, en primíparas 4,1\%, en Multíparas 2,94\%.

12 - L. A. CALKINS Kansas City.

Mortalidad fetal en presentación de pelvis es del 2 al $10 \%$.

12 - SCHMITZ HERBERT AND SMITH CHARLES Chicago, Ill.

Mortalidad fetal global en partos de pelvis es del $12,12 \%$ Mortalidad fetal corregida es del $4,12 \%$.

13 - HALL AND KOHL del Departamento de Obstetricia y 
Ginecología de la Universidad del Estado de New York, Escuela de Medicina N.Y. City.

Mortalidad fetal en Pelvis Incompleta 13,9\%.

Mortalidad fetal en Pelvis Completa 11,7\%.

14 - RAFAEL PERALTA C. La presentación de pelvis en Obstetricia. Etiología, Pronóstico y Conducta. Tesis de Grado. Bogotá, Octubre $1^{9}$ de 1940.

Frecuencia global de la mortalidad fetal: 28,51\%.

Mortalidad por retención de cabeza última: 8,51\%.

15 - DR. JESUS ALBERTO GOMEZ PALACINO. Parto en presentación de Pelvis Incompleta. Comentarios. 1956.

La mortalidad global en el parto de Pelvis Incompleta en el Instituto Materno Infantil fué de $17,3 \%$ para los fetos, en el último año.

La mortalidad depurada comprende un $5,12 \%$ de los casos estudiados.

1 - JUAN LEON. Tratado de Obstetricia:

La mortalidad fetal global no depurada es alta en algunas estadísticas ( $20 \%$ o más) ; mediana en otras $(10 \%$ a $20 \%$ ) y aún baja (menos del 10\%).

La mortalidad fetal depurada en muchas estadísticas es alta por pasar del $10 \%$, en algunos ambientes es halagüeña por estar por debajo del $2 \%$.

2 - EASTMAN EN LA OBSTETRICIA DE WILLIAMS.

La mortalidad fetal total (no corregida) es del $20 \%$.

16 - THOMPSON MORTALIDAD PERINATAL EN PRESENTACION DE PELVIS.

Del Departamento de Obstetricia y Ginecología Universidad de Indiana - Indianápolis.

La frecuencia de mortalidad perinatal en fetos de 1.000 a 2.499 grs. es de $30,2 \%$.

En fetos de 2.500 grs. o más es del 2,8\%.

No ha sido posible buscar la morbilidad perinatal por las 
mismas fallas anotadas de indisciplina en la manufactura de las historias, en este caso de los niños; ni siquiera en la mortalidad tener una seguridad absoluta de las causas de muerte, ya que solamente un feto llegó a la autopsia, o por lo menos que aparezca constancia en la Historia.

La incidencia de los partos espontáneos fué del 56,28\%, la cual compararemos con otras estadísticas :

1 - JUAN LEON. Tratado de Obstetricia:

La cifra más alta de partos espontáneos es la de Vemerlin y Ribón 60 a $70 \%$.

En nuestro servicio del Hospital de Argerich, la proporción de partos espontáneos alcanzaba a $28,1 \%$.

13 - HALL AND KOHL de la Escuela de Medicina de New York City.

La incidencia de partos espontáneos es del $20 \%$.

14 - RAFAEL PERALTA C. La presentación de pelvis en Obstetricia. Tesis de Grado.

Frecuencia del parto espontáneo $61 \%$.

La frecuencia de intervenciones cesáreas en el Instituto fué del $7,03 \%$, la cual también me permito comparar con otras estadísticas.

13 - HALL AND KOHL. Del Departamento de Obstetricia y Ginecología de la Escuela de Medicina de Ney York, City.

La frecuencia de intervención cesárea en la presentación de pelvis es del $10,7 \%$.

1 - JUAN LEON. Tratado de Obstetricia.

La incidencia de Cesáreas en la presentación pelviana fué de 2,6\% en el Hospital de Argerich.

16 - THOMPSON. "Mortalidad perinatal en presentación de Pelvis". Universidad de Indiana - Indianápolis.

La frecuencia de Intervención Cesárea en la presentación de pelvis es del $6 \%$.

5 - PROFESOR RAFAEL RAMIREZ MERCHAN. Comunicación personal. 
La incidencia de la Cesárea en la presentación de pelvis es del 13 al $45 \%$.

Sería conveniente hacer una revisión completa de los métodos empleados en el auxilio del parto de pelvis a excepción de la cesárea ya que la mortalidad presenta su mayor porcentaje en este tipo de parto; quizás con una mejor valoración de los casos y con atención de Obstetras de mayor experiencia puedan disminuírse estas cifras; posiblemente el suministro de Fórceps de Piper disminuya en algo la mortalidad fetal.

Es conveniente, también anotar que la incidencia de Cesárea podría elevarse un poco más en vista de sus benéficos resultados en especial en todos aquellos casos que surjan dudas sobre la competencia del canal pélvico en relación al tamaño del feto (apreciación que debe ser hecha siempre por el personal más experimentado de la Institucióńn), en todos los casos en que exista cualquier tipo de alteración de la contracción uterina, en especial aquellos que se acompañan de rigidez del cuello con la consiguiente demora en su dilatación.

Es también necesario, hacer énfasis sobre el uso de la radiografía en los partos de pelvis para valorar el tamaño fetal y la forma y capacidad de la pelvis materna; también auxilia este método para el diagnóstico de vicios de actitud fetal in útero, tales como deflexión primitiva de la cabeza, brazo nucal, etc. además nos permite apreciar cualquier vicio de conformación fetal como Hidrocefalias, tumores, Hidrops fetales, etc.

\section{TERCER CAPITULO}

\section{RESUMEN}

Se consultaron 597 casos de pelvis que corresponden al $2,82 \%$ de los partos en general, de los cuales el $1.828 \%$ corresponden a los partos de pelvis completa, el $0.883 \%$ a los partos en pelvis incompletas y el $0.019 \%$ a los partos en presentación de pelvis no especificadas. La mayor incidencia se registró en los partos a término con el $63,82 \%$.

En general el tipo de parto fue espontáneo siendo el 57,17\% la frecuencia del parto en pelvis Completa y el 54,01\% en pelvis Incompleta, con un promedio general de partos espontáneos en pelvis del $56,28 \%$. Para los partos auxiliados y corregidos la fre- 
cuencia fué del $32,16 \%$ para pelvis en general. Para los partos operatorios la frecuencia general fué del $7.03 \%$ para las Cesáreas y del $4,18 \%$ para las extracciones podálicas.

La mortalidad general sin depurar es del $26,74 \%$ para los partos de pelvis incompleta y del $24,87 \%$ para la pelvis completa. Depurando la mortalidad, no contando sino los casos de muerte por causas obstétricas puras, la mortalidad baja al $9,09 \%$ para la pelvis incompleta y al $6,73 \%$ para las pelvis completas. La mortalidad depurada en las Cesáreas es del $0 \%$ y en las Extracciones podálicas es del $20 \%$.

La mortalidad depurada en los partos espontáneos es del $6,55 \%$ y en los partos Auxiliados y corregidos es del 14,06\%.

\section{CUARTO CAPITULO}

\section{CONCLUSIONES}

1 - La evaluación del caso y el parto debe ser atendido por el personal más especializado del equipo.

2 - Debe procurarse el parto espontáneo e intervenir en el período expulsivo solamente cuando haya indicación precisa.

3 - Se insinúa la conveniencia de practicar sistemáticamente la episiotomía para el parto de pelvis.

4 - Deben aumentarse las intervenciones cesáreas, ya que la mortalidad fetal es nula y dan mayor seguridad en los casos dudosos.

5 - Debería instalarse la rutina de pelvimetría para mayor orientación en la conducta a seguir y hacer una valoración clínica muy detallada de la pelvis en su forma y tamaño.

6 - Es conveniente la rutinización de analgesia obstétrica para disminuír el "Stress" y las complicaciones de retención de cabeza última.

7 - Deben mejorarse las condiciones de asepsia para disminuír la morbi-mortalidad por infecciones del recién nacido.

8 - Deben limitarse las extracciones podálicas por la alta mortalidad fetal y preferirse en muchos de estos casos la cesárea. 
9 - Sería conveniente rutinizar la operación cesárea erı las primíparas añosas.

10 - Se hace necesario revaluar el uso de la versión externa en las consultas prenatales; ya que el riesgo de éstas es bastante bajo.

11 - Se impone la unificación en la terminología de los tipos de parto en presentación de pelvis para evitar confusiones posteriores y facilitar la revisión de las Historias Clínicas. Propongo la siguiente clasificación, que fué precisamente la que utilicé en este trabajo: a) Partos espontáneos, b) Partos auxiliados y corregidos, y c) Partos operatorios; en estos últimos incluímos: Cesáreas, Extracciones podálicas, aplicaciones de Fórceps para cabeza última y embriotomías.

\section{BIBLIOGRAFIA}

1 LEON J. Tratado de Obstetricia (ed. 1\%) Tomo 1: 1.064. Editorial Científica Argentina, S. R. L. Buenos Aires 1956.

2 EASTMAN, N. J. Obstetricia de Williams. (ed. 2) 822 . Unión Tipográfica. Editorial Hispano Americana, México, 1953.

3 VERMELIN, H. y RIBON, M. Enciclopedia Médico-Quirúrgica. Tomo I. Obstétrico: 5.026 A20 1952.

4 NUBEOLA-ZARATE. Tratado de Obstetricia. Tomo I: Editorial Labor S. A. Barcelona 1951.

5 RAMIREZ, M. R. Comunicación Personal, Febrero, 1961.

6 PERALTA, C. R. Comunicación Personal, Febrero. 1961.

7 LLERAS, C. S. Comunicación Personal, Febrero, 1961.

8 GOMEZ, P. J. Comunicación Personal, Febrero, 1961.

9 SANCHEZ, T. F. Comunicación Personal, Febrero, 1961.

10 SCHMITZ, H. y SMITH CH. Breech presentation, Am. J. Obst. \& Gynec. 69:984, 1955.

11 KNIGHT, R., and O'CONNELL, C. P. A ten year analysis of breech deliveries, 1939. 1948. Am. J. Obst. \& Gynec. 64:1049, 1952.

12 CALKINS, L. A. Breech presentation. Am. J. Obst. \& Gynec. 69:977, 1955.

13 HALL, J. E. and KOHL, S. Breech presentation. Am. J. Obst. \& Gynec. 72:977, 1956.

14 PERALTA, C. R. La presentación de pelvis en Obstetricia. Etiología, Pronóstico y Conducta. (Tesis de grado) 103. Elitorial Lumen, Bogotá, 1940.

15 GOMEZ, P. J. A. Parto en presentación de Pelvis Incompleta. Comentarios Revista del Hospital de San Juan de Dios. IV: 140, 1956.

16 THOMPSON, J. F. Perinatal Mortality in Breech Presentation. Obst. \& Gynec. 15:415, 1960.

17 LA ESTADISTICA DE LOS SERVICIOS DE CLINICA OBSTETRICA DEL INSTITUTO MATERNO INFANTIL DE BOGOTA DE MARZO DEL AÑO 1958 A MARZO DEL AÑO 1960. 